\title{
A SINR MAXIMIZING 2D RAKE RECEIVER FOR MULTI-SENSOR WCDMA MOBILE TERMINALS
}

\author{
Massimiliano Lenardi and Dirk T. M. Slock \\ Mobile Communications Department - Institut Eurécom * \\ 2229 Route des Crêtes, B.P. 193, F-06904 Sophia Antipolis Cedex, FRANCE \\ E-mail: $\{$ lenardi, slock\}@eurecom.fr
}

\begin{abstract}
The 2D RAKE receiver is a spatio-temporal matched filter (MF), matched to the operations of spreading, pulse-shape filtering and spatio-temporal channel filtering. An SINR maximizing linear receiver may perform much better. In the downlink, in which the channel is the same for all intracell signals, and with orthogonal codes and cell-dependent scrambling, good SINR performance can be attained with a RAKElike receiver. In particular, we replace the pulse-shape MF with another FIR filter, and the sparse propagation channel MF by another sparse filter. The FIR filter can be spatiotemporal or just temporal. In the latter case, it can be identical or different for the different antennas. The sparse filter is spatiotemporal. We compare different choices for the design of the FIR filter and the sparse filter.
\end{abstract}

\section{Introduction}

In the FDD mode of the Wideband CDMA (WCDMA) option of the 3GPP UMTS proposal for cellular wireless communications, both uplink and downlink use DS-CDMA communications. This paper focuses on the downlink, where a set of orthogonal periodic spreading sequences are used, to take advantage of the synchronicity (between users) of the downlink.

To limit interference between cells though, a cell-dependent scrambling gets added which does not destroy the orthogonality between the intracell users. Due to the scrambling (which can be considered as a stationary chip rate sequence), the received signal is cyclostationary at the chip rate. The conventional receiver for DS-CDMA communications is the RAKE receiver. The RAKE receiver is a matched filter (MF), matched to the operations of spreading, pulse shape filtering and channel filtering. Such a MF does not maximize the SINR, but only the SNR. The RAKE receiver is a restricted linear optimal receiver in the sense that it would be optimal if only the additive white noise (and not any interference) would be present.

Even though the received signal is cyclostationary at the chip rate (and not at the symbol rate, so that signal subspaces are time-varying) linear multiuser detectors (MUD) can be meaningfully applied to achieve much improved performance (SINR) over the RAKE receiver. The general linear MMSE receiver is time-varying however, due to the presence of the scrambler. For such a receiver spanning several symbol periods, the complexity for applying the filter can be quite high, due to the multiplications of signals with arbitrarily valued coefficients at chip rate. And of course, the complexity for producing the time-varying filter coefficients is enormous. From this point of view, nonlin-

\footnotetext{
*Eurécom's research is partially supported by its industrial partners: Ascom, Swisscom, Thales Communications, STMicroelectronics, CEGETEL, Motorola, France Télécom, Bouygues Telecom, Hitachi Europe Ltd. and Texas Instruments. The work leading to this paper was also partially supported by the French RNRT (National Network for Telecommunications Research) project AUBE.
}

ear Interference Cancellation (IC) such as Parallel IC (PIC), as typically applied in the uplink at the Base Station (BS), is more interesting since it consists of a cascade of RAKE reception and refiltering by the channel. And the complexity is similar between aperiodic or periodic codes. However, nonlinear approaches require a good initialization (by a linear receiver) for proper operations. And the detection of all (intracell) users at the mobile terminal seems overkill.

Now, structurally constrained linear detectors exist that show a reasonable complexity/performance trade-off. Indeed, apart from synchronicity, another characteristic of the downlink is that all intracell signals pass through the same channel (if the BS does not apply user-specific beamforming or sectoring). So, considering only the intracell interference (and not the intercell interference and noise), a receiver consisting of a zero-forcing equalizer followed by a descrambler and a correlator would be optimal (maximize SIR). Indeed, the equalizer restores orthogonality of the codes (which was destroyed by the delay spread of the multipath channel) so that a simple correlator then suffices to pick out the signal with the code of interest while perfectly suppressing all other (orthogonal) codes. Such a receiver is also suboptimal though since the zero-forcing equalizer enhances the noise and intercell interference. The RAKE maximizes the SNR while this receiver maximizes the SIR (counting the intercell interference with the noise). The performance criterion that needs to be optimized though is the SINR.

In [1] we proposed a generalized linear receiver, the maxSINR receiver, which encompasses the RAKE and the equalizerplus-correlator receivers [2] as special cases. The structure is the same of the RAKE receiver, but the channel and pulse shape matched filters are replaced by an equalizer filter that is designed to maximize the SINR at the output of the receiver. So the receiver is a cascade of a linear (short-term) time-invariant equalizer, a descrambler and a correlator. So the overall linear receiver is time-varying but the time-variation is completely concentrated in the descrambler. It turns out that the optimal design of the equalizer that leads to maximum SINR at the output of the overall receiver leads to the MMSE equalizer (with the received signal considered to be cyclostationary at chip rate). In [3] we studied different lower-complexity implementations of the equalizer, including a cascade of a pulse shape matched filter and a sparse filter, whose coefficients were optimized to maximize the output SINR.

In the case of a mobile terminal equipped with multiple sensors, the equalizer simply becomes a spatio-temporal MMSE equaliser. The multi-sensor aspect improves the equalization performance and allows to suppress similarly structured intercell interference. In this paper we study further reduced complexity versions of this max-SINR receiver, analyzing the performance and comparing it to that of the full complexity max-SINR and of the RAKE receivers. In the reduced complexity version, the MMSE equalizer gets factored (as in the RAKE) into a short 
FIR filter and a sparse filter that spans the delay spread of the channel. Filtering by the short filter needs to be carried out at chip rate, while filtering with the sparse filter is implemented by combining after the correlator (at symbol rate) as in the RAKE. Various choices are possible for the FIR filter and the sparse filter. These choices lead to a certain SINR at the output of the overall receiver. The maximization of the SINR can be transformed into a quadratic optimization problem subject to a linear constraint. For the FIR filter, the linear constraint is that the inner product with the pulse shape matched filter should be fixed. For the sparse filter, the inner product with the propagation channel matched filter should be fixed. Subject to these constraints, still various choices are possible for the filters. The adaptation of the SINR maximizing version of the receiver can be done in a semi-blind fashion at symbol rate, while requiring the same information (channel estimate) as the RAKE receiver. The complexity of the receiver gets essentially determined by the choice of either a temporal or a spatiotemporal solution for the FIR filter, by the number of coefficients in the various filters, and by the complexity of the adaptation mechanisms if any parts of the receiver get adapted.

\section{Multiuser Downlink Signal Model}

Fig. 1 shows the downlink signal model in baseband, between the mobile receiver and the main base station. Signals coming from other BSs are of the same structure, but pass through different channels, and are included in the additive noise $v(t)$. The $K$ users are assumed to transmit linearly modulated signals over the same linear multipath channel. The symbol and chip periods $T$ and $T_{c}$ are related through the spreading factor $L: T=L T_{c}$, which is assumed here to be common for all the users. The total chip sequence $b_{l}$ is the sum of the chip sequences of all the users, each one given by the product between the $n$th symbol of the $k$ th user and an aperiodic spreading sequence $w_{k, l}$ which is itself the product of a periodic Walsh-Hadamard (with unit energy) spreading sequence $\boldsymbol{c}_{k}=\left[\begin{array}{llll}\boldsymbol{c}_{k, 0} & \boldsymbol{c}_{k, 1} \cdots \boldsymbol{c}_{k, L-1}\end{array}\right]^{T}$, and a basestation specific unit magnitude complex scrambling sequence $s_{l}$ with variance $1, w_{k, l}=c_{k, l} \bmod { }_{L} s_{l}$ :

$$
b_{l}=\sum_{k=1}^{K} b_{k, l}=\sum_{k=1}^{K} a_{k,\left\lfloor\frac{l}{L}\right\rfloor} w_{k, l} .
$$

The chip sequence $b_{l}$ gets transformed into a continuous-time signal by filtering it with the pulse shape $p(t)$ and then passes through the multipath propagation channel $h(t)$ to yield the received signal $y(t)$. The receiver samples $M$ times per chip the lowpass filtered received signal.

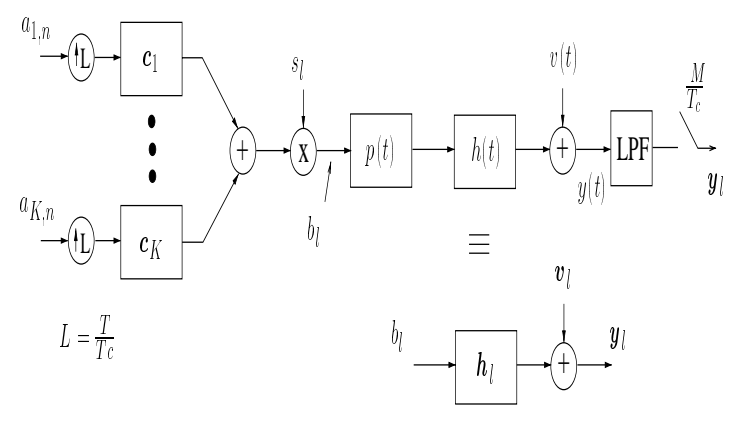

Figure 1. Downlink signal model BS-MS

Stacking the $M$ samples per chip period in vectors, we get for the sampled received signal at MS antenna $j$ during chip period $l$

$$
\boldsymbol{y}_{l}^{j}=\sum_{k=1}^{K} \sum_{i=0}^{N-1} h_{i}^{j} b_{k, l-i}+\boldsymbol{v}_{l}^{j}
$$

where

$$
\boldsymbol{y}_{l}^{j}=\left[\begin{array}{c}
y_{1, l}^{j} \\
\vdots \\
y_{M, l}^{j}
\end{array}\right], h_{l}^{j}=\left[\begin{array}{c}
h_{1, l}^{j} \\
\vdots \\
h_{M, l}^{j}
\end{array}\right], \boldsymbol{v}_{l}^{j}=\left[\begin{array}{c}
v_{1, l}^{j} \\
\vdots \\
v_{M, l}^{j}
\end{array}\right]
$$

Here $\boldsymbol{y}_{l}^{j}$ and $\boldsymbol{h}_{l}^{j}$ represent the vectors at the chip rate of samples at the sampling rate. $h_{l}^{j}$ denotes the overall channel, including pulse shape, propagation channel and receiver filter for the MS antenna $j(j=1 \cdots J)$. The overall channel is assumed to have a delay spread of $N$ chips due to contributions from $P$ paths. The multipath description of the channel for oversampling phase $m$ at antenna $j$ and during chip period $l$ is

$$
h_{m, l}^{j}=\sum_{p=1}^{P} \alpha_{p}^{j} p\left(l T_{c}+\frac{(m-1) T_{c}}{M}-\tau_{p}\right)
$$

For antenna $j,\left\{\alpha_{p}^{j}\right\}$ is the complex amplitude of path $p$ with corresponding delay $\left\{\tau_{p}\right\}$ (the delays for a given path are equal for all $J$ MS antennas). If we model the scrambling sequence and the symbol sequences as independent i.i.d. sequences, then the chip sequence $b_{l}$ is a sum of $K$ independent white noises (chip rate i.i.d. sequences, hence stationary). The intracell contribution to $\boldsymbol{y}_{l}^{j}$ then is a stationary (vector) process (the continuous-time counterpart is cyclostationary with chip period). The intercell interference is a sum of contributions that are of the same form as the intracell contribution. The remaining noise is assumed to be white stationary noise. Hence the sum of intercell interference and noise, $v_{l}^{j}$, is stationary. In the case of multiple MS antennas, the total received signal from a BS is

$$
\boldsymbol{y}_{l}=\left[\left(\boldsymbol{y}_{l}^{1}\right)^{T} \cdots\left(\boldsymbol{y}_{l}^{J}\right)^{T}\right]^{T}
$$

where $e_{j}$ is a unit vector of size $J$ with a 1 in position $j$. The total channel is then $h_{l}=\left[\left(\boldsymbol{h}_{l}^{1}\right)^{T} \cdots\left(\boldsymbol{h}_{l}^{J}\right)^{T}\right]^{T}$.

\section{Max-SINR Receiver Structure}

As shown in Fig. 2, the receiver is constrained to be a chip rate filter $f$ followed by a descrambler and a correlator with the spreading code of the user of interest, which is here assumed to be user 1 . So the receiver has the same structure as a RAKE receiver, except that the channel matched filter gets replaced by a general filter $f$. If a sparse (path-wise) representation is used for the channel, then the channel matched filter leads to a RAKE structure with one finger per path. In Fig. 2, the operation "S/P" denotes a serial to parallel conversion which stacks the $L$ most recent inputs into a vector. The correlator can also be viewed as a matched filter, matched to the spreading code filter, but here it is simply depicted as an inner product on a downsampled vectorized signal.

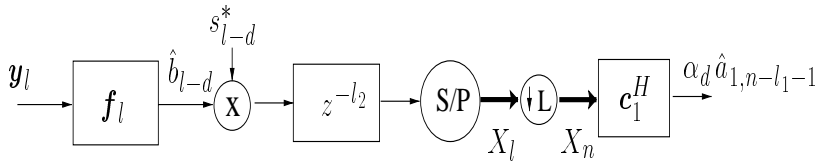

Figure 2. The downlink receiver structure

While the RAKE is one particular instance of the proposed receiver structure, another special case is the equalizer receiver. To describe this case more precisely, let $h(z)=\sum_{l=0}^{N-1} h_{l} z^{-l}$ be the $M J \times 1$ FIR channel transfer function and $f(z)=$ $\sum_{l=0}^{I-1} f_{l} z^{-l}$ the $1 \times M J$ FIR filter transfer function of length $I$ chips. The cascade of channel and filter gives $f(z) h(z)=$ 
$\sum_{l=0}^{I+N-2} \alpha_{l} z^{-l}=\alpha(z)$. In particular, for a zero-forcing (ZF) equalizer with a delay of $d$ chips, we get $f(z) h(z)=z^{-d}$. In general, we can write for the filter-channel cascade

$$
\mathcal{T}(f) \mathcal{T}(h)=\mathcal{T}(\boldsymbol{\alpha})=\mathcal{T}\left(\boldsymbol{\alpha}_{d}\right)+\mathcal{T}\left(\overline{\boldsymbol{\alpha}_{d}}\right)
$$

where $\mathcal{T}($.) is a block Toeplitz filtering matrix and

$$
\begin{aligned}
& \boldsymbol{\alpha}=\left[\alpha_{0} \cdots \alpha_{I+N-2}\right], \boldsymbol{\alpha}_{d}=\left[0 \cdots 0 \alpha_{d} 0 \cdots 0\right]
\end{aligned}
$$

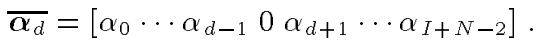

In the noiseless case (and no intercell interference), the use of a ZF equalizer leads to $\overline{\boldsymbol{\alpha}_{d}}=[0 \cdots 0]$ and $\widehat{a}_{1, n}=a_{1, n}\left(\alpha_{d}=1\right)$. A RAKE receiver corresponds to $f=h^{H}, \alpha_{d}=\|\boldsymbol{h}\|^{2}, I=N$, where $h=\left[h_{N-1}^{T} \cdots h_{0}^{T}\right]^{T}$.

The analysis done in [1] shows that, due to the orthogonality of the spreading codes and to the i.i.d. character of the scrambler, the SINR, $\Gamma$, at the receiver output is

$$
\Gamma=\frac{\sigma_{1}^{2}\left|\alpha_{d}\right|^{2}}{f R_{V V} \boldsymbol{f}^{H}+\left.\sigma_{t o t}^{2}|| \overline{\boldsymbol{\alpha}_{d}}\right|^{2}}=\frac{\sigma_{1}^{2}\left|\alpha_{d}\right|^{2}}{f R_{Y Y} f^{H}-\sigma_{t o t}^{2}\left|\alpha_{d}\right|^{2}}
$$

where $\sigma_{k}^{2}=\mathrm{E}\left|a_{k, n}\right|^{2}, \sigma_{\text {tot }}^{2}=\frac{1}{L} \sum_{k=1}^{K} \sigma_{k}^{2}$ and $R_{Y Y}=R_{V V}+\sigma_{\text {tot }}^{2} \mathcal{T}\left(h^{\prime}\right) \mathcal{T}^{H}\left(h^{\prime}\right)$ where $\mathcal{T}\left(h^{\prime}\right)$ denotes a block Toeplitz convolution matrix and $h^{\prime}$ denotes $h$ but with its chip rate coefficients in reversed order. The choice for the filter $f$ that leads to maximum receiver output SINR is unique up to a scale factor and can be found as the solution to the following problem

$$
\begin{aligned}
\boldsymbol{f}_{M A X} & =\arg \max _{\boldsymbol{f}: \boldsymbol{f} h=1} \Gamma=\arg \min _{\boldsymbol{f}: \boldsymbol{f} h=1} f R_{Y Y} \boldsymbol{f}^{H} \\
\Rightarrow \boldsymbol{f}_{M A X} & =\left(\boldsymbol{h}^{H} R_{Y Y}^{-1} \boldsymbol{h}\right)^{-1} h^{H} R_{Y Y}^{-1}
\end{aligned}
$$

The maximum SINR becomes $\left(\alpha_{d}^{M A X}=1\right)$

$$
\Gamma_{M A X}=\frac{\sigma_{1}^{2}}{\left(\boldsymbol{h}^{H} R_{Y Y}^{-1} \boldsymbol{h}\right)^{-1}-\sigma_{\text {tot }}^{2}}
$$

As pointed out in [1], this receiver corresponds to the cascade of an (unbiased if $\alpha_{d}=1$ ) MMSE receiver for the desired user's chip sequence, followed by a descrambler and a correlator. In the noiseless case, the MMSE receiver $f_{M A X}$ becomes a ZF equalizer. In fact, the max-SINR receiver is related to the linear MMSE receiver which is

$$
\widehat{a}_{1}=R_{a_{1} Y} R_{Y Y}^{-1} Y=\sigma_{a}^{2} \boldsymbol{c}_{1}^{H} S^{H} \mathcal{T}^{H}(h) R_{Y Y}^{-1} Y
$$

where we omitted time indices and we assume that the FIR LMMSE is based on a stretch of signal $Y . S$ is a diagonal matrix containing the scrambling sequence over a certain symbol period, $R_{a_{1} Y}=\mathrm{E}_{a, v} a_{1} Y^{H}, R_{Y Y}=\mathrm{E}_{a, v} Y Y^{H}$ where $\mathrm{E}_{a, v}$ denotes expectation over symbols and noise. Due to the scrambling sequence, $R_{Y Y}$ is time-varying and the whole LMMSE is time-varying. The max-SINR is obtained when $R_{Y Y}$ is computed using $\mathrm{E}_{a, v, s}$, so by averaging over the scrambler also, considered as an i.i.d. sequence. In that case $\sigma_{a}^{2} \mathcal{T}^{H}(h) R_{Y Y}^{-1}$ is of the form $\mathcal{T}(f)$ where $f$ is an MMSE (chip rate) equalizer. So the LMMSE receiver becomes a MMSE equalizer-descramblercorrelator cascade.

\section{Path-Wise Receiver Structures}

The equalizer filter $f_{M A X}$ presented in the previous section replaces at the same time the pulse shape and the channel matched filters, leaving complete freedom to the optimization process.
Other possibilities arise when we impose a particular structure on the receiver. We shall here focus on structured equalizers that are the cascade of a short spatiotemporal FIR filter followed by a sparse spatiotemporal filter. The RAKE is a particular instance of this structure, with the FIR filter being the pulse shape matched filter per antenna, and the sparse filter being the 2D matched filter to the 2D sparse propagation channel. We shall here consider several choices for the short FIR filter, with the sparse filter portion being optimized for max SINR. The constrained receiver structure considered here is depicted in Fig. 3.

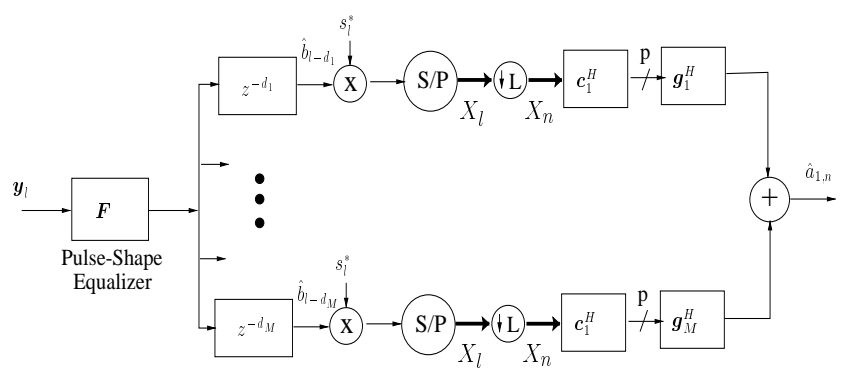

Figure 3. The path-wise equalizer RAKE structure

\section{IV.1. Pulse-Shape MF (GRAKE)}

The pulse shape adopted by the 3G UMTS norm is a root raised cosine (RRC) with roll-off 0.22 . In a first instance, we shall take the short FIR filter to simply be the pulse shape matched filter, as in the RAKE receiver. However, the sparse filter coefficients are optimized for maximum SINR. This receiver structure was introduced in [4] as the GRAKE and independently in [3]. To analyze this receiver structure, we can write the overall channel $h$ in (4) as

$$
h=\mathcal{T}^{H}\left(\boldsymbol{p} \otimes I_{J}\right) \boldsymbol{h}_{p r o p}=\mathcal{P}_{s p}^{H} \boldsymbol{h}_{s p}
$$

where $\mathcal{T}^{H}\left(\boldsymbol{p} \otimes I_{J}\right)$ is the convolution matrix of the root raised cosine $p(t)$ and $h_{\text {prop }}$ is the vector of samples of the (sparse) multipath propagation channel (MPC). Due to the sparseness of the MPC (train of pulses), $\mathcal{T}^{H}\left(p \otimes I_{J}\right)$ can be reduced to $\mathcal{P}_{s p}^{H}$ (selected columns) and $h_{\text {prop }}$ to $h_{s p}$ (the non-zero coefficients of $h_{\text {prop }}$ ). The receiver filter is then factored into a RRC matched filter (represented by a convolution matrix as $\mathcal{T}^{H}\left(p \otimes I_{J}\right)$ or $\left.\mathcal{P}_{s p}\right)$ and an optimized (sparse) filter:

$$
f=g_{\text {prop }} \mathcal{T}\left(\boldsymbol{p} \otimes I_{J}\right)=g_{s p} \mathcal{P}_{s p}
$$

Optimizing the coefficients of the spatiotemporal (temporally) sparse filter $g_{s p}$ for maximum SINR at the output of the overall receiver, we get for the SINR

$$
\Gamma_{G R A K E}=\frac{\sigma_{1}^{2}}{\left(h^{H} \mathcal{P}_{s p}^{H}\left(\mathcal{P}_{s p} R_{Y Y} \mathcal{P}_{s p}^{H}\right)^{-1} \mathcal{P}_{s p} h\right)^{-1}-\sigma_{\text {tot }}^{2}}
$$

For improved performance, the tap positions in $g_{\text {prop }}$ do not necessarily correspond to the taps in the propagation channel $h_{\text {prop }}$. Also, increasing the number of taps beyond the number of paths (such that more than one tap per path is available) will obviously improve performance, but at the cost of an increase in complexity. In the GRAKE, the channel impulse response as seen at the input of the sparse filter is the channel impulse response filtered by the pulse shape matched filter, or hence the sparse propagation channel filtered by the pulse shape correlation sequence. The sampled pulse shape correlation sequence would be a delta function if no oversampling would be used. In the case of oversampling however, it contains a number of nonzero samples. Hence, putting more than one tap per finger in the sparse filter of the receiver will improve performance. 


\section{IV.2. Path-Wise Equalizer (PWEQRAKE)}

In the unconstrained equalizer-correlator receiver, the optimal equalizer $f_{M A X}$ is essentially a MMSE equalizer $h^{H} R_{Y Y}^{-1}=$ $h_{\text {prop }}^{H} \mathcal{T}\left(\boldsymbol{p} \otimes I_{J}\right) R_{Y Y}^{-1}$. Hence, a logical choice for the short FIR filter in the path-wise structured equalizer would be a pathwise equalizer, or hence a MMSE pulse shape equalizer (corresponding to $\left.\left(p \otimes I_{J}\right) R_{Y Y}^{-1}\right)$. The resulting receiver is still depicted in Fig. 3. One may remark that in that case, the channel as seen at the input of the sparse filter is the cascade of the channel impulse response and the pulse shape equalizer and hence also the cascade of the sparse propagation channel and the equalized pulse shape (cascade of the pulse shape and its MMSE equalizer). This equalized pulse shape should have approximately one significant nonzero coefficient. Hence, the sparse filter with one tap per path appears to be well adapted in this case.

The spatiotemporal FIR MMSE equalizer for the pulse shape is of the form

$$
F=\left(p \otimes I_{J}\right) R_{Y Y}^{-1}
$$

where $R_{Y Y}$ now is the covariance matrix spanning $2 Q+1$ chip periods, and $p$ contains $2 Q+1$ blocks of size $M \times M$, that are filled with the $M$ (oversampling) phases of the pulse shape (matched filter) in the time span $(-Q, Q)$ chip periods. The resulting $F$ contains $M J$ rows, each row corresponding to the pulse shape equalizer for a particular sampling phase and a particular antenna. The receiver filter (overall equalizer) is now again factored into a short FIR filter, being the pulse shape equalizer, and an optimized (sparse) filter:

$$
f=g_{\text {prop }} \mathcal{T}(F)=g_{s p} \mathcal{F}_{s p} .
$$

Optimizing the coefficients of the spatiotemporal (temporally) sparse filter $g_{s p}$ for maximum SINR at the output of the overall receiver, we get for the SINR

$$
\Gamma_{P W E Q R A K E}=\frac{\sigma_{1}^{2}}{\left(\boldsymbol{h}^{H} \mathcal{F}_{s p}^{H}\left(\mathcal{F}_{s p} R_{Y Y} \mathcal{F}_{s p}^{H}\right)^{-1} \mathcal{F}_{s p} \boldsymbol{h}\right)^{-1}-\sigma_{t o t}^{2}}
$$

\section{IV.3. Averaged PWEQRAKE (APWEQRAKE) and per-} antenna equalizer (PAEQRAKE)

The spatiotemporal MMSE equalization of the pulse shape leads to nonnegligible complexity unless $Q$ is kept very small. To simplify the equalization operation, we can average the RX signal covariance matrix over the antennas to obtain $\bar{R}_{Y Y}$ with which we construct a temporal pulse shape equalizer

$$
\bar{F}=p \bar{R}_{Y Y}^{-1}
$$

We then apply this temporal equalizer to each antenna signal, hence

$$
F=\bar{F} \otimes I_{J} .
$$

The SINR for the APWEQRAKE can be obtained by substituting in (16) and (17) the temporal pulse shape equalizer $F$ in (19).

An alternative strategy would be to have an optimized temporal equalizer per antenna instead of an averaged one, that is we take

$$
\bar{F}_{j}=p \bar{R}_{Y_{j} Y_{j}}^{-1}=\left[\bar{F}_{j, Q} \cdots \bar{F}_{j, Q}\right]
$$

and $F=\left[F^{(Q)} \ldots F^{(-Q)}\right]$ where $F^{(q)}$ is constructed from $\left\{\bar{F}_{j, q}\right\}_{j=1}^{p}$. The SINR for the per-antenna equalizer (PAEQRAKE) can be, again, obtained by substituting $F$ in (16) and (17).

\section{IV.4. Joint Iterative Equalizer (JIEQRAKE)}

The best structurally constrained receiver can be obtained by optimizing both the FIR and the sparse filters for maximum SINR. This can be done by alternating optimizations. Initializing $F$ as the pulse-shape matched filter, we can indeed optimize the coefficients of the sparse spatiotemporal filter, as in the GRAKE receiver, subject to the linear constraint $\boldsymbol{g}_{s p} \boldsymbol{h}_{s p}=1$. Then we can maximize the SINR w.r.t. the FIR filter $F$. We shall take for $F$ a different temporal filter for each antenna (so not a spatiotemporal filter). The constraint on each of these temporal FIR filters (for the different antennas) is that the inner product with the pulse shape matched filter should be one. Then we can reoptimize $g_{s p}$ and so on until convergence. Eqs. (9) and (16) tell us how to implement each one of these iterations, where firstly $\boldsymbol{g}_{s p}$ and secondly $F$ are optimized. The SINR for the joint-iterative equalizer (JIEQRAKE) can be, again, obtained by substituting expressions of $\boldsymbol{g}_{s p}$ and $F$ in (16) and using Eq. (8) $\left(\alpha_{d}=1\right)$.

\section{Simulations}

Various simulations with different sets of parameters have been performed. All the $K$ users are considered synchronous and use the same spreading factor. The FIR channel is the convolution of a sparse channel with 4 (environment 3 ) or 8 taps (environment 6, two separated clusters of 4 taps each) and a pulse shape (root-raised cosine with roll-off factor of 0.22). The UMTS chip rate $(3.84 \mathrm{Mchips} / \mathrm{sec})$ is assumed, leading to a maximum overall channel length of $N=12$ chips for environment 3 and of $N=20$ chips for environment 6 . An oversampling factor of $M=2$ or $M=4$ is used in the simulations and it is indicated in the title of each figure. The spreading factor (SF) is 32 and there are 9 intracell users per active BS. All intracell and intercell interferers have the same power as the user of interest.

The figures show the performance of various receiver instances in terms of the output signal-to-interference-and-noise (SINR) ratios versus the SNR at the receiver when the complexity for all the FIR filters is comparable. In the figure legends, "RAKE" refers to the performance of the RAKE receiver (continuous line), "Unc. max-SINR" to the uncostrained max-SINR receiver of section III (dashed line), "G-RAKE" to the receiver of section IV.1 (dotted line), "PWeq-RAKE" to the path-wise equalizer of section IV.2 (star line), "Avg PWeq-RAKE" and "PAeqRAKE" to the receivers of section IV.3 (dashed-dotted and dashed-dotted-diamond lines respectively) and "JIeq-RAKE" to the Joint Iterative receiver of section IV.4 (dashed-star line).

In the cases of $1 \mathrm{MS}$ antenna and 1 or 2 BS transmitting (Fig. 4 and Fig. 5), all the path-wise receivers easily outperform the RAKE, with the GRAKE receiver performing generally worse then the other path-wise structures. We can notice that all the considered structures saturate for high input SNR, due to the fact that there are not enough degrees of freedom to obtain complete cancellation of the intracell and/or intercell interference (zeroforcing).

When 2 MS antennas are implemented (Fig. 6 and Fig. 7), zero-forcing is possible for the unconstrained max-SINR receiver as well as for the JIeqRAKE (the high-SNR zero-forcing asymptote is not visible on the SNR scale of Fig. 6). The other receiver structures perform similarly, with the GRAKE being the best. When 3 MS antennas are implemented and 2 BS are transmitting (Fig. 8 and Fig. 9), we have similar performance for all the receivers.

\section{Conclusions}

We have introduced constrained linear receiver structures for the MS, consisting of a cascade of an FIR filter, a sparse filter, a 
descrambler and a correlator. When the two filters are optimized jointly, performance gets close to that of the Max-SINR receiver with one unconstrained filter. There is no SINR saturation for the Max-SINR when the number of MS antennas is at least equal to the number of BS, but when we just have equality, the distance between the low SNR and high SNR asymptotes of the SINR curve can get large.

\section{References}

[1] M. Lenardi and D. T. M. Slock, "A RAKE Receiver with Intracell Interference Cancellation for a DS-CDMA Synchronous Downlink with Orthogonal Codes," in Proc. VTC 2000, (Tokyo, Japan), May 2000.

[2] I. Ghauri and D. T. M. Slock, "Linear receivers for the DSCDMA downlink exploiting orthogonality of spreading sequences," in Proc. 32nd Asilomar Conf. on Signals, Systems \& Computers, (Pacific Grove, CA), November 1998.

[3] M. Lenardi and D. T. M. Slock, "SINR Maximizing Equalizer Receiver for DS-CDMA," in Proc. EUSIPCO 2000, (Tampere, Finland), September 2000.

[4] T. O. G. Bottomley and Y. Wang, "A generalized rake receiver for interference suppression," IEEE Journal Selected Areas Communications, vol. 18, pp. 1536-1545, August 2000.

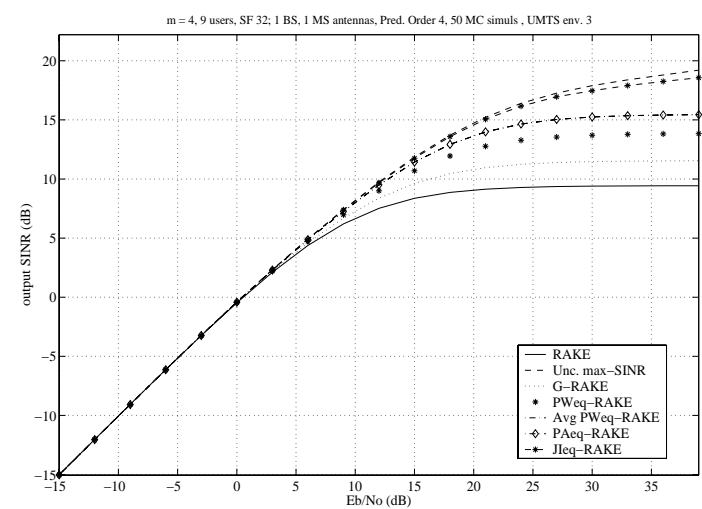

Figure 4. Theoretical output SINR versus SNR, 1 MS antenna and 1 trasmitting BS

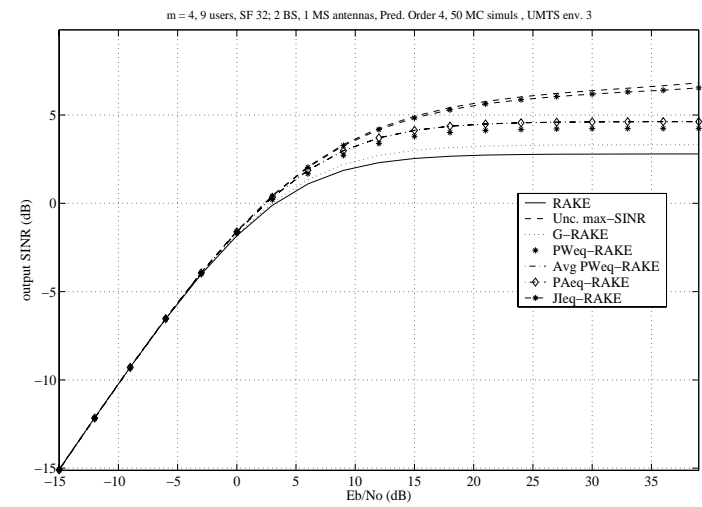

Figure 5. Theoretical output SINR versus SNR, $1 \mathrm{MS}$ antenna and 2 trasmitting BS

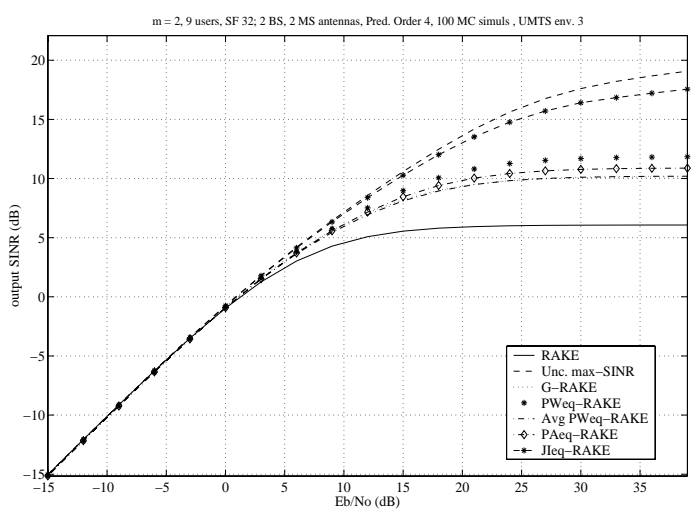

Figure 6. Theoretical output SINR versus SNR, 2 MS antenna and 2 trasmitting BS

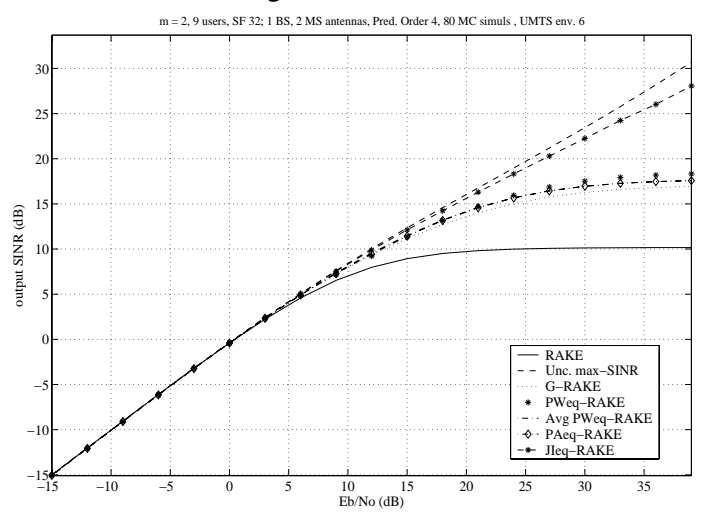

Figure 7. Theoretical output SINR versus SNR, 2 MS antenna and 1 trasmitting BS

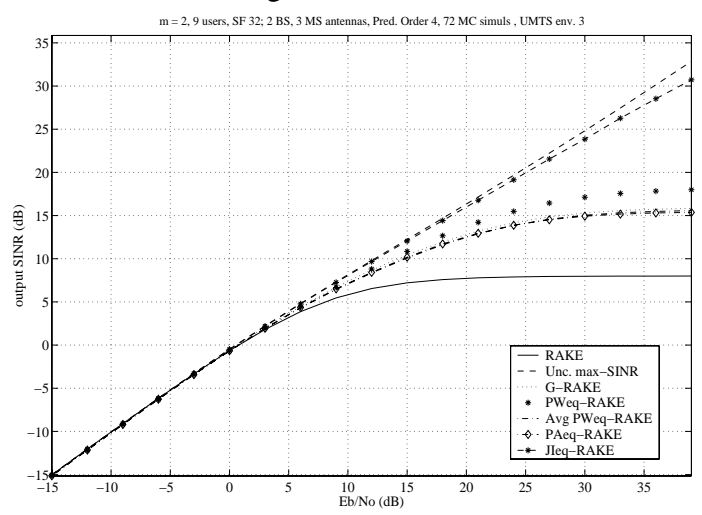

Figure 8. Theoretical output SINR versus SNR, 3 MS antenna and 2 trasmitting BS

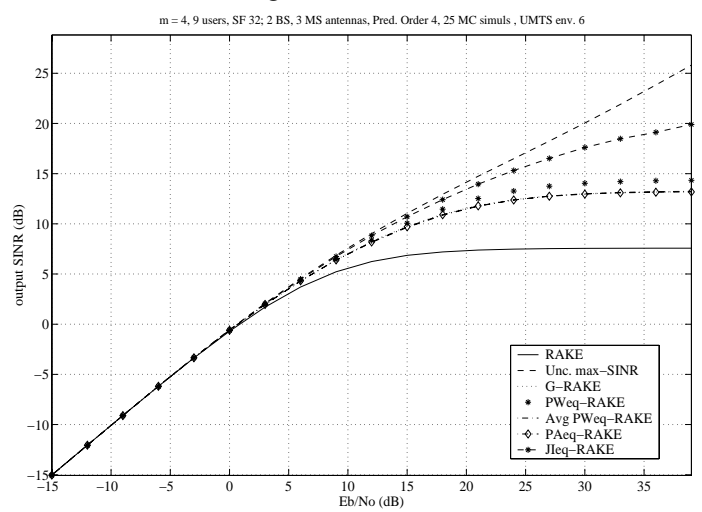

Figure 9. Theoretical output SINR versus SNR, 3 MS antenna and 2 trasmitting BS 